\title{
Propuesta pedagógica constructivista para la enseñanza de literatura española, en $2^{\circ}$ año de enseñanza media
}

\author{
Marisa Guzmán Munita*, \\ Mario Díaz Díaz ${ }^{\star \star}$
}

\section{RESUMEN}

Un desafío para los profesores de castellano en Chile corresponde a la vinculación de los contenidos teóricos con la elaboración de actividades prácticas, que permitan el acercamiento de los estudiantes a diversas temáticas, desde el enfoque constructivista que plantea el currículum nacional. El presente artículo tiene por objetivo proveer de una propuesta pedagógica, que asume este enfoque al orientar metodológicamente la enseñanza de la Literatura Española. Se repara, particularmente, en la poesía ascética medieval, en correspondencia con lo prescrito por el programa de estudio de la asignatura para segundo año de Enseñanza Media.

Palabras clave: Literatura Española, poesía ascética, propuesta pedagógica, constructivismo, segundo año de Enseñanza Media.

\section{Constructivist pedagogical proposal for the teaching of Spanish Literature in the second grade of High School} \begin{abstract}
A challenge for teachers of Spanish language in Chile corresponds to linking the theoretical content with the development of practical activities that allow students to approach various topics, from the constructivist approach imposed by the national curriculum. This article aims to provide an educational proposal assuming this approach and methodologically guiding the teaching of Spanish Literature. It particularly emphasizes the ascetic medieval poetry in correspondence with the requirements for the curriculum of the course for the second grade of secondary school.
\end{abstract}

Key words: Spanish Literature, ascetic poetry, pedagogical approach, constructivism, second grade of secondary School.

* Marisa Guzmán Munita es Doctora en Ciencias Pedagógicas por la Universidad Varona de La Habana y Coordinadora de Prácticas Profesionales de la Escuela de Educación en Castellano de la Universidad Católica Silva Henríquez (UCSH). E-mail: joournalistmgm@gmail.com

Mario Díaz Díaz es estudiante de tercer año de Licenciatura en Castellano de la Universidad de Santiago (USACH).E-mail: mario.diaz@usach.cl 


\section{Introducción}

Desde la reforma educacional de los años '90, los esfuerzos han apuntado a mejorar la calidad y equidad de la educación, asumiéndose un enfoque constructivista para la enseñanza en todos los niveles (MINEDUC, 20IIa). No obstante, pese a la relevancia que ha adquirido el constructivismo en el currículum chileno, su aplicación en el aula es aún limitada, lo que afecta la calidad de la educación provista (MINEDUC, 2005).

En este sentido, un reto para la consecución de las aspiraciones curriculares, lo impone la apertura del propio profesor por incrementar su capital docente pedagógico a objeto de responder con propiedad a los desafíos que impone el ejercicio docente constructivista y su propia superación (Coll, I996; 2000). La necesidad de exhibir la consistencia teórica y práctica que el proceso de enseñanza-aprendizaje requiere en su globalidad, motiva la propuesta de una alternativa pedagógica, como una manera de invitar a los profesores de castellano a visualizar formas análogas de ejecutar y controlar el desarrollo de las habilidades, actitudes y valores que subyacen en los contenidos mismos.

Desde esta perspectiva, la propuesta pedagógica constituye la vía para avanzar a la "(..) autorregulación, compromiso y responsabilidad social, como elemento básico de las motivaciones profesionales e identidad, con la carrera y la profesión" (Olivares \& Mariño, 2007: I I). Así, la gestión pedagógica del proceso de enseñanza-aprendizaje, que conduce el profesor en el aula, debe propiciar oportunidades de compartir y contrastar habilidades (saber hacer), capacidades (saber ser) y competencias (saber hacer con otros), a fin de acompañar al estudiante en la construcción y descubrimiento de conocimientos; implementando estrategias acordes a sus intereses y características socioculturales; creando un clima de aula de respeto, confianza y afecto; y usando el error que emana de la construcción de esquemas e imágenes, como mecanismos para profundizar los aprendizajes.

La propuesta pedagógica que se presenta plantea un camino para organizar y orientar el trabajo pedagógico relacionado con la 
enseñanza de la Literatura Española Medieval, y se sitúa en el ámbito de la poesía ascética. Tal propuesta, grafica el enfoque metodológico asumido para propiciar que el conocimiento del canon de lecturas asociadas a la producción artística del período barroco español se incremente y desarrolle mediante un proceso de construcción y reconstrucción sucesivo, progresivo y flexible. El enfoque constructivista orienta procedimientos, selecciona medios materiales y virtuales, aporta estrategias y sugiere formas de evaluación, ilustrando lo que puede hacerse en una clase en que se aborde una figura representativa del canon literario español: Fray Luis de León (I527-I59I).

\section{La literatura española en segundo año de Enseñanza Media}

A partir de la actualización del marco curricular realizada el año 2009, los Objetivos Generales y Contenidos Mínimos han sido traducidos en Aprendizajes Esperados (AE) e Indicadores de Evaluación, los que -en conjunto- establecen los logros que deben exhibir los estudiantes al término del proceso parcial (la unidad temática) y global (el año lectivo). En el caso de la asignatura de Lenguaje, en segundo año de enseñana media, el programa establece el desarrollo de habilidades y competencias a través de cuatro unidades temáticas: narrativa, drama, textos no literarios y poesía.

Asimismo, el programa releva la importancia de integrar a los diversos contenidos, los Objetivos Fundamentales Transversales (OFT) definidos en el currículum, por cuanto apuntan al desarrollo personal, ético, social e intelectual de los educandos, los que no se logran por medio de una asignatura en particular, sino mediante el esfuerzo conjunto de otras áreas del conocimiento.

Respecto de la labor del profesor en las aulas, MINEDUC (20I Ia: 34) señala:

Las habilidades deben trabajarse a través de los objetivos de aprendizaje de cada asignatura. Si bien estos Objetivos en sí suponen el desarrollo de actividades, el docente puede lograr distintos niveles cognitivos al cruzar distintas habilidades, 
lo que permite profundizar, variar, establecer relaciones $y$ reforzar el desarrollo del pensamiento. En síntesis, sobre un mismo conocimiento es posible desarrollar distintas operaciones mentales de diverso nivel, dando calidad al aprendizaje a lograr.

La revisión del programa de segundo año de enseñanza media, permite advertir que dentro de las lecturas fundamentales sugeridas para abordar la IV Unidad temática: "Poesía", se mencionan autores como Francisco de Quevedo, Garcilaso de la Vega, Alonso de Ercilla, entre otros, haciéndose, también, mención al poeta foco de este trabajo: Fray Luis de León. Este autor es uno de los escritores más importantes de la segunda fase del Renacimiento español, siendo representante de la literatura ascética. Esta corriente destaca en su producción literaria los esfuerzos que el espíritu debe realizar para alcanzar la perfección moral, aconsejando sobre los caminos que conducen a la salvación divina. Aunque se reconoce que esta temática no resulta especialmente motivadora en el ámbito escolar contemporáneo, el carácter polémico de este monje agustino, catedrático de la Universidad de Salamanca -que llegó a estar cinco años detenido por desacato a una orden del Concilio de Trento- aporta un caudal interesante de posibilidades de profundizar en el contexto en que se elabora su obra "Los nombres de Cristo".

En consideración que los planes y programas establecen que la enseñanza de la poesía debe conducir al desarrollo de conocimientos, habilidades y actitudes, la propuesta pedagógica que se presenta considera los objetivos fundamentales transversales (OFT) establecidos para orientar el sentido y la resignificación que cada alumno construya en torno a los conocimientos conceptuales presentados. De esta manera, el desarrollo de las actitudes y valores que persiguen consolidar los OFT a través de sus cinco ámbitos, tienen presencia en la propuesta pedagógica, la que orienta el cómo vincular los conocimientos teóricos constitutivos de cada Programa de estudio, con actividades pedagógicas constructivistas, a objeto de lograr tanto la comprensión y esclarecimiento de los contenidos conceptuales primordiales del tema enseñado, como la adquisición 
y desarrollo de las habilidades y competencias requeridas para asimilarlos, más allá de lo memorístico o anecdótico.

\section{El diseño metodológico de la propuesta}

La presente propuesta pedagógica considera la vinculación de la teoría con la práctica, en su diseño metodológico, el que se explica en una clase de dos horas pedagógicas (90 minutos) para la que se aporta una actividad pedagógica relacionada con la poesía ascética de Fray Luis de León, específicamente, de su poema "De los nombres de Cristo”. Para orientar las acciones de los profesores, se sugiere la siguiente lógica:

a. Alineación del aprendizaje esperado (AE) con el objetivo fundamental transversal (OFT) correspondiente al contenido e indicadores de evaluación, para articular decisiones intencionadas y coherentes, en la planificación del trayecto del proceso de enseñanza-aprendizaje.

b. Descripción de las acciones que realizan los estudiantes, en torno a los tres momentos fundamentales de la clase que media el profesor: introducción, desarrollo y cierre.

c. Para la etapa de Inicio, se sugiere que el docente implemente estrategias de motivación, diversas y plausibles, en el contexto sociocultural de los estudiantes de segundo año de enseñanza media a los que se dirija.

d. Para el Desarrollo de la clase, se propone una metodología activoparticipativa, que enfatice el intercambio dialógico y medie la construcción conjunta del conocimiento con actividades que relacionen las problemáticas que plantea Fray Luis de León, con el contexto contemporáneo, a la luz de los propios sentimientos, experiencias, valores y creencias de los alumnos.

e. En correspondencia con lo anterior, el Cierre evalúa la coherencia de las actividades con los $\mathrm{AE}$ proyectados para la misma mediante actividades de indagación crítica y debate.

De los ocho aprendizajes esperados (AE) (tres de lectura, tres de escritura y dos de comunicación oral), prescritos para abordar la 
unidad de Poesía, la propuesta pedagógica selecciona el siguiente: AE 02 Lectura: Interpretar poemas comentados en clases.

- Reflexiona sobre aspectos temáticos y de contenido.

- Relaciona los poemas con el contexto de producción.

El AE 02 permite establecer la relación con el siguiente OFT:

- Desarrollo de habilidades comunicativas y de análisis, interpretación y sintesis de la información.

- Profundiza sus conocimientos y muestra interés por los temas que se discuten en clases.

- Comparte sus interpretaciones, posturas y criticas sobre los textos leídos.

- Muestra interés frente a los comentarios y reflexiones del resto de sus compañeros y el profesor.

En relación a los indicadores de evaluación, los estudiantes:

- Explican el tema del poema identificando la postura desde la cual ha sido abordado.

- Relacionan los sentimientos, conflictos y temas expresados en los textos leidos, con su experiencia personal, valores, creencias $y$ prejuicios.

- Explican la influencia del contexto sociocultural de producción en el tratamiento del tema abordado en los textos leídos.

Lo anterior se vincula con el siguiente: Contenido conceptual

- Análisis e interpretación del poema "De los nombres de Cristo" de Fray Luis de León.

\section{Propuesta pedagógica}

A continuación, se orientan los procedimientos y se exhibe una muestra de los medios materiales y virtuales posibles de emplear para gestionar estrategias y formas de evaluación, que desde una perspectiva constructivista, permitan abordar la figura de Fray Luis de León en segundo año de enseñanza media. 


\section{Inicio: Motivación}

Indicaciones al docente: La lectura de poemas debe ser una oportunidad para que los estudiantes exploren las amplias posibilidades que ofrece el lenguaje, para exponer temas, expresar sentimientos y asumir posiciones, desde amplias perspectivas. De esta forma, el comentario en clases del poema "De los nombres de Cristo" debe conllevar a la reflexión de los estudiantes en torno a los aspectos de forma (estilo, recursos) y contenido (temática, postura del hablante lírico), en vinculación con su contexto histórico, político y cultural de producción. Para ello, es importante que el docente sea creativo al relacionar la obra de Fray Luis de León con el momento histórico cultural actual, a fin de conducir a los estudiantes a develar la pertinencia de este autor en cuanto a la expresión de temáticas aún vigentes.

En este sentido, el pizarrón constituye un excelente recurso material para estructurar la secuencia que ha de seguir la clase, sugiriéndose explicitar en este-desde los inicios- un Título de la sesión que llame la atención de los estudiantes, a objeto de, posteriormente, indicar el Objetivo de la misma.

Motivación: El contenido asociado a la literatura ascética exige emplear estrategias de motivación a fin de despertar la atención y curiosidad que conlleve a los estudiantes a involucrarse activamente con los $\mathrm{AE}$ desde el inicio de la clase. Por lo anterior, se sugieren las siguientes acciones y estrategias para este momento:

Título de clase creativo: "Fray Luis de León: Un revolucionario de su época". Conflicto cognitivo: Para generar el conflicto cognitivo, el profesor escribe con letras de gran tamaño una pregunta detonadora, que tenga vinculación con la temática del contenido a enseñar, a la figura del autor y al contexto social en que se originó su poesía. Una pregunta, por ejemplo, sería la siguiente:

¿Es posible alejarse del mundo material para tener un mayor desarrollo espiritual?

Lluvia de ideas: Resulta indispensable que el docente recoja los aprendizajes previos (¿qué saben/conocen...?) de los tópicos a 
abordar (el Barroco y su contexto histórico, del autor, del término 'ascética' propiamente tal, de la poesía española...), estimulando la participación activa y el respeto por los turnos de intervención. Tras mediar la síntesis de los aportes, se aclaran los tópicos introductorios y se escribe el objetivo de la clase.

Objetivo de la clase: Reflexionar sobre la temática, contenido y contexto de producción de la poesía ascética española.

\section{Desarrollo: las actividades}

Para avanzar a esta etapa, se sugieren las siguientes estrategias:

El vínculo con la vida cotidiana: Durante la exposición de contenidos, resulta vital la relación del contenido abordado con la vida cotidiana, con énfasis en llevar la reflexión a dimensionar que la comprensión del pasado ayuda a entender el mundo que nos rodea y constituye el fundamento para ampliar visiones que conduzcan al desarrollo personal e intelectual.

Dato anecdótico del poeta: Para generar un mayor interés de parte de los estudiantes hacia el contenido a abordar durante la clase, se puede hacer referencia a una anécdota vivida por Fray Luis de León a propósito de que este autor permaneció cinco años encerrado en la cárcel, procesado por la Inquisición a causa de ciertas sospechas en materia de fe. Comentarle a los alumnos que al cabo de estos cinco años y, luego, de probar su inocencia, Fray Luis de León, fue puesto en libertad y volvió a dictar cátedra en la Universidad de Salamanca. En la primera clase que realizó, comenzó con la frase: "Como decíamos ayer...", tal como acostumbraba a recapitular lo que había explicado el día anterior, pero ahora con cinco años de diferencia. El profesor puede emplear esta anécdota para capturar el interés de los estudiantes en distintos momentos, por ejemplo, entrando a la sala de clases y diciendo la frase: "Como decíamos ayer... ", para después continuar comentando su biografía.

En virtud del $A E$ "Interpretar poemas comentados en clases", el contenido "Análisis e interpretación del poema "De los nombres de Cristo" y el objetivo de la clase "Reflexionar sobre la temática, 
contenido y contexto de producción de la poesía ascética española”, las acciones antecedentes se complementarán con el uso de un recurso multimedia. Mediante este recurso virtual, los estudiantes se introducen en la corriente ascética y el contexto socio-histórico que la enmarca, empleando un video de 3 minutos seleccionado de la web, que mezcla lectura, música e imágenes, para mostrar los rasgos biográficos más relevantes del autor y su contexto ${ }^{3}$.

Tras el video, los estudiantes comentan la incidencia de la Inquisición en el contexto histórico, y realizan una analogía con formas contemporáneas de censura (religiosas, sociales, políticas, étnicas...). Se distribuyen fragmentos distintos del poema "De los nombres de Cristo" (en el anexo se exhiben ejemplos) y se procede a su lectura; lectura que el profesor modela con cuidado énfasis en reflejar el estado anímico del hablante lírico.

Se divide al curso en ocho equipos de trabajo y se propone el análisis e interpretación del poema a partir de responder una Guía de apoyo con las siguientes preguntas:

- ¿Cuál es la actitud del hablante lírico?, ¿cómo es su temple de ánimo? Justifícalo con un ejemplo.

- ¿De qué manera influye el contexto de producción en el ritmo que le imprime Fray Luis al poema? Comenta cómo lo infieres.

- ¿Cuál es el mensaje del poema?

- ¿Qué recursos literarios y discursivos emplea el hablante para manifestar sus ideas? Menciónalos.

- ¿De qué manera se relacionan los sentimientos y tema expresado, con tu propia experiencia personal?

Luego de socializar oralmente el análisis por equipos, el profesor media un plenario donde los alumnos discuten el título de la clase a la luz de la temática, contenido y contexto del autor, y responden: ¿es posible afirmar que Fray Luis de León fue un revolucionario de su época?

Ver video "Fray Luis de León: Biografía" disponible en http://www.youtube.com/ watch?v=tZfyW7 wue-Q 


\section{Cierre: evaluación}

En el cierre, se recoge el objetivo de la clase mediante preguntas, por ejemplo: ¿qué aprendimos hoy...? El profesor vincula las principales conclusiones y orienta la reflexión y el debate respecto a valorar la contribución de la poesía ascética española para plantear disquisiciones existenciales, que permiten ampliar su análisis a problemáticas contemporáneas.

A continuación, se fundamentan las decisiones metodológicas adoptadas para articular la propuesta presentada.

\section{Decisiones metodológicas}

En correspondencia con el enfoque constructivista que tiene la propuesta, la variedad de recursos sugeridos para conducir la clase: uso del pizarrón, material concreto (guía, selección de fragmentos del poema) y material virtual (video con música ad boc) facilitan la inmersión activa del estudiante en la temática abordada. Tales recursos resultan coherentes con las estrategias metodológicas aportadas, que median la interacción con énfasis en la participación de los estudiantes. Así, lo revelan las preguntas iniciales; el empleo de la pedagogía del humor para explicar datos anecdóticos; la inclusión de ejemplos contextualizados a la edad e intereses de los estudiantes y la vinculación del conocimiento con la vida cotidiana. Al mismo tiempo, la decisión de dividir al curso por equipos (lo que bien podría hacerse desde considerar agruparlos por diversos estilos de aprendizaje) enriquece el análisis e interpretación de la obra de Fray Luis de León, desde diversas perspectivas.

Desde el punto de vista comunicativo, la propuesta busca desmarcarse de las viejas prácticas pedagógicas -centradas en una enseñanza vertical y reproductiva- para dar protagonismo al estudiante, buscando desarrollar el intercambio dialógico y debate en torno a la influencia del contexto sociocultural de producción de las obras, en el tratamiento de los tópicos que abordan y la vigencia de dichos temas. 
Las actividades planteadas consideran el desarrollo de los tres ejes propuestos por las bases curriculares: lectura, escritura y comunicación oral, donde el profesor asume un rol de mediador, estimulando la activa disposición que precisa la construcción del conocimiento. Se estima que las diversas estrategias, recursos y métodos que conllevan situar esta propuesta, permiten sostener que podría constituirse en un aporte para llevar al aula la temática de la poesía ascética religiosa en la figura de Fray Luis de León y su obra; desde un enfoque constructivista y crítico, el cual releva la discusión de los elementos valóricos relacionados con los contenidos por cuanto son, precisamente, los que le otorgan sentido y relevancia al aprendizaje.

\section{Conclusiones}

Los procedimientos, métodos y estrategias que provee la propuesta pedagógica presentada, orientan el desarrollo de babilidades comunicativas mediante el intercambio, discusión y debate, así como a través de la exposición oral y escrita del producto del análisis, interpretación y síntesis de la información sobre la temática abordada, que reciben los alumnos.

No obstante, resulta especialmente relevante que el profesor genere un clima de aula de respeto (por los turnos de intervención, por la tolerancia a las ideas ajenas), que permita, efectivamente, compartir interpretaciones, posturas y críticas sobre los textos leídos.

Desde esta perspectiva, se considera que la motivación de los estudiantes por sus aprendizajes, depende en gran medida del liderazgo que el profesor asuma al conducir el proceso y del capital pedagógico que demuestre para hacerlo.

Un docente con plena conciencia del encargo social que implica, no solo instruye, sino que educa; revisa permanentemente sus prácticas pedagógicas, a fin de integrar los procedimientos y recursos necesarios que le permitan vincular los conocimientos conceptuales, con las actitudes y valores que se ponen en juego para concretarlo.

De esta manera, si bien la propuesta pedagógica aporta luces respecto de cómo concretar el enfoque constructivista en el aula, esta u otras 
alternativas resultan insostenibles sin la reflexión activa y crítica de los propios profesores respecto de sí mismos y de su actuación con otros, para sortear, positivamente, los desafíos que implica conducir el proceso de enseñanza-aprendizaje desde este enfoque.

\section{Referencias bibliográficas}

Coll, C. (1996). Constructivismo y educación escolar: Ni hablamos siempre de lo mismo ni lohacemos desde la misma postura epistemológica. Anuario de Psicología, 69, I53-178.

Coll, C. (2000). Constructivismo e intervención educativa: cómo enseñar lo que ha de construirse. En Barbera, E. El constructivismo en la práctica (p. I57). Caracas: Laboratorio Educativo.

De León, F. L. (1999). De los nombres de Cristo. Recuperado de: http://www. cervantesvirtual.com/obra-visor/de-los-nombres-de-cristo--2/html/ fedb9fc0-82bI-IIdf-acc7-002I85ce6064_I.html\#I_2_

MINEDUC (2005). Informe comisión sobre formación inicial docente. Recuperado de: http://www.oei.es/pdfs/info_formacion_inicial_docente_chile.pdf

(20IIa). Fundamentos bases curriculares. Propuesta presentada para la aprobación del Consejo Nacional de Educación. Unidad de Currículum y Evaluación. Santiago: Autor.

(20IIb). Programa de estudio de segundo año medio Lenguaje y Comunicación. Santiago: Autor.

(2012). Evaluación inicia. Presentación de resultados 2012.

Recuperado de: http://www.mineduc.cl/usuarios/mineduc/ doc/201308221629I00.RESULTADOS_EVALUACION_IN ICIA.pdf

Olivares, E. \& Mariño, J. (2007). La motivación profesional pedagógica: necesidad y realidad. Congreso Internacional Pedagogía 2007, La Habana, Cuba. 


\section{Anexos}

Fragmentos del poema "De los nombres de Cristo"

"Y, a la verdad, los poetas antiguos, y cuanto más antiguos tanto con mayor cuidado, atendieron mucho a huir de lo lascivo y artificioso, de que está lleno el amor que en las ciudades se cría, que tiene poco de verdad, y mucho de arte y de torpeza. Mas el pastoril, como tienen los pastores los ánimos sencillos y no contaminados con vicios, es puro y ordenado a buen fin; y como gozan del sosiego y libertad de negocios que les ofrece la vida sola del campo, no habiendo en él cosa que los divierta, es muy vivo y agudo. Y ayúdales a ello también la vista desembarazada, de que continuo gozan, del cielo y de la tierra y de los demás elementos; que es ella en sí una imagen clara, o por mejor decir, una como escuela de amor puro y verdadero. Porque los demuestra a todos amistados entre sí y puestos en orden, y abrazados, como si dijésemos, unos con otros, y concertados con armonía grandísima, y respondiéndose a veces, y comunicándose sus virtudes, y pasándose unos en otros y ayuntándose y mezclándose todos, y con su mezcla y ayuntamiento sacando de continuo a luz y produciendo los frutos que hermosean el aire y la tierra. Así que los pastores son en esto aventajados a los otros hombres. Y así, sea esta la segunda cosa que señalamos en la condición del Pastor; que es muy dispuesto al bien querer".

(Cap. Pastor, párr. 5).

2.- "Y porque, Juliano, los caminos son en diferentes maneras, que unos son llanos y abiertos, y otros estrechos y de cuesta, y unos más largos, y otros que son como sendas de atajo; Cristo, verdadero camino y universal, cuanto es de su parte, contiene todas estas diferencias en sí; que tiene llanezas abiertas y sin dificultad de tropiezos, por donde caminan descansadamente los flacos, y tiene sendas más estrechas y altas para los que son de más fuerza, y tiene rodeos para unos, porque así les conviene, y ni más ni menos por donde atajen y abrevien los que se quisieren apresurar. Mas veamos lo que escribe de este 
nuestro camino Isaías: "Y habrá allí senda y camino, y será llamado camino santo. No caminará por él persona no limpia, y será derecho este camino para vosotros; los ignorantes en él no se perderán. No habrá león en él, ni bestia fiera, ni subirá por él ninguna mala alimaña. Caminarle han los librados, y los redimidos por el Señor volverán, y vendrán a Sión con loores y gozo sobre sus cabezas sin fin. Ellos asirán del gozo y de la alegría, y el dolor y el gemido huirá de ellos”.

(Cap. Camino, párr. II).

3.- "Y sea la tercera lo que toca a su oficio, que aunque es oficio de gobernar y regir, pero es muy diferente de los otros gobiernos. Porque lo uno, su gobierno no consiste en dar leyes ni en poner mandamientos, sino en apacentar y alimentar a los que gobierna. Y lo segundo, no guarda una regla generalmente con todos y en todos los tiempos, sino en cada tiempo y en cada ocasión ordena su gobierno conforme al caso particular del que rige. Lo tercero, no es gobierno el suyo que se reparte y ejercita por muchos ministros, sino él solo administra todo lo que a su grey le conviene; que él la apasta y la abreva, y la baña y la trasquila, y la cura y la castiga, y la reposa y la recrea $y$ hace música, y la ampara y defiende. Y últimamente, es propio de su oficio recoger lo esparcido y traer a un rebaño a muchos, que de suyo cada uno de ellos caminara por sí. Por donde las sagradas Letras, de lo esparcido y descarriado y perdido dicen siempre que son como ovejas que no tienen pastor; como en San Mateo se ve y en libro de los Reyes y en otros lugares. De manera que la vida del pastor es inocente y sosegada y deleitosa, y la condición de su estado es inclinada al amor, y su ejercicio es gobernar dando pasto, y acomodando su gobierno a las condiciones particulares de cada uno, y siendo él solo para los que gobierna todo lo que le es necesario, y enderezando siempre su obra a esto, que es hacer rebaño y grey”.

(Cap. Pastor, párr. 5). 